\title{
A Stochastic Growth Model for Cancer Cells under Mutation and Metastasis in an Organ
}

\author{
Jayabharathiraj, J. \\ Department of Statistics, Pondicherry University, India \\ jayabharathi8@gmail.com
}

\begin{abstract}
Keywords: Stochastic Modelling, Birth and Death Processes, Differential Equations, Cancer Cell Growth.
\end{abstract}

\begin{abstract}
This study has proposed a stochastic model for cancerous growth due to its metastasis in an organ. The birth-and-death and migration processes based on growth and loss rates of pathogenesis of malignant and normal cells are considered. It is assumed that the growth and loss/death rates of both normal and malignant cells follows Poisson processes. The joint probability generating functions in the form of partial differential equation along with statistical measures were derived in terms of system of ordinary differential equations. The Model behaviour was analysed by solving those differential equations and presented graphically.
\end{abstract}

\section{Introduction}

The carcinogenesis is a complex random multistep process involving initiation, promotion and progression of cells (Tan, 1989, 1987). Transforming normal cells into cancerous cells in any organ is usually initiated with simple mutation and aggravate with abnormal proliferation. Carcinogenesis is highly complex and stochastic, the mutation processes of normal to intermediate stage and intermediate to malignant stage cells are purely random in nature (Serio, 1984). The cancer cell growth has involved more than one stage. While transforming as a malignant cell, it may undergoes birth-and-death process and two mutation processes. It is explained in term of qualitative and epidemiological phenomena through two stage stochastic model (Quinn (1997), Moolvagkar and Venzon (1979), Birkhead (1986), Moolgavkar (1983)) and their objective of carcinogenesis studies are more concerned on finding incidence functions (hazard function).

Rao et al., (2014) have studied the growth of cancer cells in the presents of migration and cells growth in the secondary location. The statistical moments are derived explicitly based on homogeneous processes with boundary and initial conditions. The developed model is not solved to a solution so that to obtain the survival function of the cells. In this paper, the above developed model is considered and solved to get the solution to the model to obtain the survival function which in turn to arrive incidence function (Birkhead, 1986). Moreover, the model has the explicit moments obtained from the system of differential equations. Thus, the systems of ordinary differential equations are solved simultaneously using numerical differential equation and presented graphically. Further, same model is extended and regression model approach is attempted to deduce the model to find the behaviour of malignant cells with the assumption of cells growths are deterministic and exponential. The results are presented graphically.

\section{Stochastic Model for Growth of Cancerous Cell}

Let $g_{11}(t)$ be the growth rate of normal cells in primary tumor; $g_{21}(t)$ be the growth rate of malignant cells in primary tumor; $g_{32}(t)$ be the growth rate of immigrant malignant cells in the secondary tumor; $d_{11}(t)$ be the loss/death rate of normal cells in primary tumor; $d_{21}(t)$ be the loss/death rate of malignant cells in primary tumor ; $\mathrm{d}_{32}(\mathrm{t})$ be the loss/death rate of malignant cells in secondary tumor; $\tau_{11}(\mathrm{t})$ be the transformation rate of normal cells to malignant cells in a primary tumor; $\tau_{21}(\mathrm{t})$ be the migration rate of malignant cells in a primary tumor to the secondary tumour; $\tau_{32}(t)$ be the emigration rate of malignant cells in the secondary tumour to other part of the body. 
The mechanisms involved in the cell divisions are purely stochastic in nature. Events occurred in non-overlapping interval of time are statistically independent are assumed.

Furthermore the following hypotheses are assumed for the model.

In a infinitesimal time $\Delta \mathrm{t}$, probability of growth of a normal cell during $\Delta \mathrm{t}$ be $\operatorname{ng}_{11}(\mathrm{t}) \Delta \mathrm{t}+\mathrm{o}(\Delta \mathrm{t})$ there exist $\mathrm{n}$ number of normal cells epoch time $\mathrm{t}$; probability of growth of a malignant cell during $\Delta \mathrm{t}$ be $\mathrm{mg}_{21}(\mathrm{t}) \Delta \mathrm{t}+\mathrm{o}(\Delta \mathrm{t})$ there exist $\mathrm{m}$ number of malignant cells epoch time $\mathrm{t}$; probability of growth of a malignant cell during $\Delta \mathrm{t}$ be $\mathrm{g}_{32}(\mathrm{t}) \Delta \mathrm{t}+\mathrm{o}(\Delta \mathrm{t})$ there exist m number of malignant cells epoch time $\mathrm{t}$; probability of death of one normal cell during $\Delta \mathrm{t}$ be $\mathrm{nd}_{11}(\mathrm{t}) \Delta \mathrm{t}+\mathrm{o}(\Delta \mathrm{t})$ there exist $\mathrm{n}$ number of normal cells there exist $n$ number of normal cells epoch time $t$; probability of death of one malignant cell during $\Delta \mathrm{t}$ be $\mathrm{md}_{21}(\mathrm{t}) \Delta \mathrm{t}+\mathrm{o}(\Delta \mathrm{t})$ there exist $\mathrm{n}$ number of normal cells there exist $\mathrm{m}$ number of malignant cells epoch time t; probability of death of immigrant malignant cell during $\Delta \mathrm{t}$ be $\mathrm{d}_{32}(\mathrm{t}) \Delta \mathrm{t}+\mathrm{o}(\Delta \mathrm{t})$; probability of a normal cell transform into malignant cells during $\Delta \mathrm{t}$ be $\mathrm{n} \tau_{11}(\mathrm{t}) \Delta \mathrm{t}+\mathrm{o}(\Delta \mathrm{t})$ there exist $\mathrm{n}$ number of normal cells; probability of a malignant cell migrate to secondary location during $\Delta \mathrm{t}$ be $\mathrm{m} \tau_{21}(\mathrm{t}) \Delta \mathrm{t}+\mathrm{o}(\Delta \mathrm{t})$ there exist m number of malignant cells; probability of a immigrant malignant cell migrate to some other location of body during $\Delta \mathrm{t}$ be $\tau_{32}(\mathrm{t}) \Delta \mathrm{t}+\mathrm{o}(\Delta \mathrm{t})$; probability of occurrence of an event other than above said events is $\mathrm{o}(\Delta \mathrm{t})^{2}$.

Let $\{\mathrm{X}(\mathrm{t}), \mathrm{t} \geq 0\}$ be the stochastic counting process of normal cells mechanism and $\{\mathrm{Y}(\mathrm{t}), \mathrm{t} \geq 0\}$ be the stochastic counting process of malignant cells mechanism. Let $\{[X(t), Y(t)], t \geq 0\}$ be a joint bivariate stochastic processes of individual stochastic processes of $\{\mathrm{X}(\mathrm{t}), \mathrm{t} \geq 0\}$ and $\{\mathrm{Y}(\mathrm{t}), \mathrm{t} \geq 0\}$. Such that, $\mathrm{P}\{[\mathrm{X}(\mathrm{t}), \mathrm{Y}(\mathrm{t})]=[\mathrm{n}, \mathrm{m}]\}=\mathrm{p}_{\mathrm{n}, \mathrm{m}}(\mathrm{t})$ and marginal processes are $\mathrm{P}\{\mathrm{X}(\mathrm{t})=\mathrm{n}\}=\mathrm{p}_{\mathrm{n}}(\mathrm{t}), \mathrm{P}\{\mathrm{Y}(\mathrm{t})=\mathrm{m}\}=\mathrm{p}_{\mathrm{m}}(\mathrm{t})$.

Let $p_{n, m}(t, t+\Delta t)$ be the probability that occurrence of one of the possible cell mechanism in an infinitesimal interval $\Delta t$, provided there exists ' $n$ ' number of normal cells and ' $m$ ' number of malignant cells in the organ epoch time' $t$ '. Then the differential - difference equations of the model (Bharucha-Reid, 1960) are,

$$
\begin{aligned}
& \begin{aligned}
\frac{d p_{n, m}(t)}{d t}=-\{ & \left.n\left(g_{11}(t)+\tau_{11}(t)+d_{11}(t)\right)+m\left(g_{21}(t)+d_{21}(t)+\tau_{21}(t)\right)+\left(g_{32}(t)+d_{32}(t)+\tau_{32}(t)\right)\right\} p_{n, m}(t) \\
& +(n-1) g_{11}(t) p_{n-1, m}(t)+(n+1) \tau_{11}(t) p_{n+1, m-1}(t)+(m-1) g_{21}(t) p_{n, m-1}(t) \\
& +(n+1) d_{11}(t) p_{n+1, m}(t)+\left\{(m+1)\left(d_{21}(t)+\tau_{21}(t)\right)+d_{32}(t)+\tau_{32}(t)\right\} p_{n, m+1}(t) \\
& +g_{32}(t) p_{n, m-1}(t) \quad
\end{aligned} \\
& \begin{aligned}
\frac{d p_{0,1}(t)}{d t}=-\left[g_{21}(t)+g_{32}(t)+d_{21}(t)+d_{32}(t)+\tau_{32}(t)\right] p_{0,1}(t)+d_{11}(t) p_{1,1}(t)+\tau_{11}(t) p_{1,0}(t) \\
+\left\{2\left(d_{21}(t)+\tau_{21}(t)\right)+d_{32}(t)+\tau_{32}(t)\right\} p_{0,2}(t)+g_{32}(t) p_{0,0}(t)
\end{aligned} \\
& \begin{aligned}
\frac{d p_{1,0}(t)}{d t}=-\left\{g_{11}(t)+\tau_{11}(t)+g_{32}(t)+d_{11}(t)+\tau_{21}(t)+d_{32}(t)+\tau_{32}(t)\right\} p_{1,0}(t)+2 d_{11}(t) p_{2,0}(t) \\
+\left\{d_{11}(t)+\tau_{21}(t)+d_{32}(t)+\tau_{32}(t)\right\} p_{1,1}(t)
\end{aligned} \\
& \frac{d p_{0,0}(t)}{d t}=-\left(g_{32}(t)+d_{32}(t)+\tau_{32}(t)\right) p_{0,0}(t)+d_{11}(t) p_{1,0}(t)+\left\{d_{11}(t)+\tau_{21}(t)+d_{32}(t)+\tau_{32}(t)\right\} p_{0,1}(t)
\end{aligned}
$$


Further,

$$
\begin{aligned}
& \frac{\mathrm{dp}_{\mathrm{n}, 0}(\mathrm{t})}{\mathrm{dt}}=-\left\{\mathrm{n}\left(\mathrm{g}_{11}(\mathrm{t})+\tau_{11}(\mathrm{t})+\mathrm{d}_{11}(\mathrm{t})\right)+\left(\mathrm{g}_{32}(\mathrm{t})+\mathrm{d}_{32}(\mathrm{t})+\tau_{32}(\mathrm{t})\right)\right\} \mathrm{p}_{\mathrm{n}, 0}(\mathrm{t}) \\
& +(n-1) g_{11}(t) p_{n-1,0}(t)+(n+1) d_{11}(t) p_{n+1,}(t) \\
& +\left\{\left(d_{21}(t)+\tau_{21}(t)\right)+d_{32}(t)+\tau_{32}(t)\right\} p_{n, 1}(t) \quad \text { for } n>1 \\
& \frac{\mathrm{dp}_{0, \mathrm{~m}}(\mathrm{t})}{\mathrm{dt}}=-\left\{\mathrm{m}\left(\mathrm{g}_{21}(\mathrm{t})+\mathrm{d}_{21}(\mathrm{t})+\tau_{21}(\mathrm{t})\right)+\left(\mathrm{g}_{32}(\mathrm{t})+\mathrm{d}_{32}(\mathrm{t})+\tau_{32}(\mathrm{t})\right)\right\} \mathrm{p}_{0, \mathrm{~m}}(\mathrm{t}) \\
& +\tau_{11}(\mathrm{t}) \mathrm{p}_{1, \mathrm{~m}-1}(\mathrm{t})+(\mathrm{m}-1) \mathrm{g}_{21}(\mathrm{t}) \mathrm{p}_{0, \mathrm{~m}-1}(\mathrm{t}) \\
& +\mathrm{d}_{11}(\mathrm{t}) \mathrm{p}_{1, \mathrm{~m}}(\mathrm{t})+\left\{(\mathrm{m}+1)\left(\mathrm{d}_{21}(\mathrm{t})+\tau_{21}(\mathrm{t})\right)+\mathrm{d}_{32}(\mathrm{t})+\tau_{32}(\mathrm{t})\right\} \mathrm{p}_{0, \mathrm{~m}+1}(\mathrm{t}) \\
& +\mathrm{g}_{32}(\mathrm{t}) \mathrm{p}_{0, \mathrm{~m}-1}(\mathrm{t}) \\
& \text { for } \mathrm{m}>1
\end{aligned}
$$

with the initial condition $\mathrm{p}_{\mathrm{N}_{0}, \mathrm{M}_{0}}(0)=1, \mathrm{p}_{\mathrm{i}, \mathrm{j}}(0)=0 \quad \forall \mathrm{i} \neq \mathrm{N}_{0} ; \mathrm{j} \neq \mathrm{M}_{0}$.

where $\mathrm{N}_{0} \& \mathrm{M}_{0}$ are initial number of normal and malignant cells respectively.

Let us introduce the joint probability generating function $\phi(x, y ; t)$ of $p_{n, m}(t)$ be $\phi(\mathrm{x}, \mathrm{y} ; \mathrm{t})=\sum_{\mathrm{m}=0}^{\infty} \sum_{\mathrm{n}=0}^{\infty} \mathrm{x}^{\mathrm{n}} \mathrm{y}^{\mathrm{m}} \mathrm{p}_{\mathrm{n}, \mathrm{m}}(\mathrm{t})$ converges for $|\mathrm{x}|<1,|\mathrm{y}|<1$. Multiplying the above differentialdifference equations from (1) to (4) with $x^{n} y^{m}$ and summing over $n, m$, we get

$$
\begin{aligned}
\frac{d \phi(x, y ; t)}{d t}=-\left(g_{11}(t)\right. & \left.+\tau_{11}(t)+d_{11}(t)\right) \sum_{m=0}^{\infty} \sum_{n=0}^{\infty} n x^{n} y^{m} p_{n, m}(t) \\
& -\left(g_{21}(t)+d_{21}(t)+\tau_{21}(t)\right) \sum_{m=0}^{\infty} \sum_{n=0}^{\infty} m x^{n} y^{m} p_{n, m}(t) \\
& -\left(g_{32}(t)+d_{32}(t)+\tau_{32}(t)\right) \sum_{m=0}^{\infty} \sum_{n=0}^{\infty} x^{n} y^{m} p_{n, m}(t) \\
& +g_{11}(t) \sum_{m=0}^{\infty} \sum_{n=0}^{\infty}(n-1) x^{n} y^{m} p_{n-1, m}(t) \\
& +\tau_{11}(t) \sum_{m=0}^{\infty} \sum_{n=0}^{\infty}(n+1) x^{n} y^{m} p_{n+1, m-1}(t) \\
& +g_{21}(t) \sum_{m=0}^{\infty} \sum_{n=0}^{\infty}(m-1) x^{n} y^{m} p_{n, m-1}(t) \\
& +d_{11}(t) \sum_{m=0}^{\infty} \sum_{n=0}^{\infty}(n+1) x^{n} y^{m} p_{n+1, m}(t) \\
& +\left(d_{21}(t)+\tau_{21}(t)\right) \sum_{m=0}^{\infty} \sum_{n=0}^{\infty}(m+1) x^{n} y^{m} p_{n, m+1}(t) \\
& +\left(d_{32}(t)+\tau_{32}(t)\right) \sum_{m=0}^{\infty} \sum_{n=0}^{\infty} x^{n} y^{m} p_{n, m+1}(t) \\
& +g_{32}(t) \sum_{m=0}^{\infty} \sum_{n=0}^{\infty} x^{n} y^{m} p_{n, m-1}(t)
\end{aligned}
$$

On simplification we obtain

$$
\begin{aligned}
\frac{\partial \phi(\mathrm{x}, \mathrm{y} ; \mathrm{t})}{\partial \mathrm{t}}=\left\{-\left(\mathrm{g}_{11}(\mathrm{t})\right.\right. & \left.\left.+\tau_{11}(\mathrm{t})+\mathrm{d}_{11}(\mathrm{t})\right) \mathrm{x}+\mathrm{d}_{11}(\mathrm{t})+\mathrm{g}_{11} \mathrm{x}^{2}+\tau_{11}(\mathrm{t}) \mathrm{y}\right\} \frac{\partial \phi(\mathrm{x}, \mathrm{y} ; \mathrm{t})}{\partial \mathrm{x}} \\
+ & \left\{-\left(\mathrm{g}_{21}(\mathrm{t})+\mathrm{d}_{21}(\mathrm{t})+\tau_{21}(\mathrm{t})\right) \mathrm{y}+\mathrm{g}_{21}(\mathrm{t}) \mathrm{y}^{2}+\left(\mathrm{d}_{21}(\mathrm{t})+\tau_{21}(\mathrm{t})\right)\right\} \frac{\partial \phi(\mathrm{x}, \mathrm{y} ; \mathrm{t})}{\partial \mathrm{y}} \\
+ & \left\{-\left(\mathrm{g}_{32}(\mathrm{t})+\mathrm{d}_{32}(\mathrm{t})+\tau_{32}(\mathrm{t})\right)+\left(\mathrm{d}_{32}(\mathrm{t})+\tau_{32}(\mathrm{t})\right) \mathrm{y}^{-1}+\mathrm{g}_{32}(\mathrm{t}) \mathrm{y}\right\} \phi(\mathrm{x}, \mathrm{y} ; \mathrm{t})
\end{aligned}
$$

where

$$
\begin{aligned}
& \frac{\partial}{\partial x} \phi(x, y ; t)=\sum_{m=0}^{\infty} \sum_{n=0}^{\infty} n x^{n-1} y^{m} p_{n, m}(t)=\sum_{m=0}^{\infty} \sum_{n=0}^{\infty}(n-1) x^{n-2} y^{m} p_{n-1, m}(t) \\
& \frac{\partial}{\partial y} \phi(x, y ; t)=\sum_{m=0}^{\infty} \sum_{n=0}^{\infty} m x^{n} y^{m-1} p_{n, m}(t)=\sum_{m=0}^{\infty} \sum_{n=0}^{\infty}(m-1) x^{n} y^{m-2} p_{n, m-1}(t)
\end{aligned}
$$


The above equation (8) is to be solved using the initial condition $\phi(x, y ; 0)=x^{N_{0}} y^{M_{0}}$ when $\mathrm{x}(0)=\mathrm{N}_{0}$ and $\mathrm{y}(0)=\mathrm{M}_{0}$. We can obtain the characteristics of the model using joint cumulant generating function of $\mathrm{p}_{\mathrm{n}, \mathrm{m}}(\mathrm{t})$. By taking $\mathrm{x}=\mathrm{e}^{\mathrm{u}}, \mathrm{y}=\mathrm{e}^{\mathrm{v}}$ and denoting $\mathrm{K}(\mathrm{u}, \mathrm{v} ; \mathrm{t})$ as the joint cumulant generating function of $\mathrm{p}_{\mathrm{n}, \mathrm{m}}(\mathrm{t})$ we get the following expression

$$
\begin{aligned}
\frac{\partial \mathrm{K}(\mathrm{u}, \mathrm{v} ; \mathrm{t})}{\partial \mathrm{t}}=\left\{-\left(\mathrm{g}_{11}(\mathrm{t})\right.\right. & \left.\left.+\tau_{11}(\mathrm{t})+\mathrm{d}_{11}(\mathrm{t})\right)+\mathrm{d}_{11}(\mathrm{t})+\mathrm{g}_{11}(\mathrm{t}) \mathrm{e}^{\mathrm{u}}+\tau_{11}(\mathrm{t}) \mathrm{e}^{\mathrm{v}-\mathrm{u}}\right\} \frac{\partial \mathrm{K}(\mathrm{u}, \mathrm{v} ; \mathrm{t})}{\partial \mathrm{u}} \\
& +\left(-\left(\mathrm{g}_{21}(\mathrm{t})+\mathrm{d}_{21}(\mathrm{t})+\tau_{21}(\mathrm{t})\right)+\mathrm{g}_{21}(\mathrm{t}) \mathrm{e}^{\mathrm{v}}+\left(\mathrm{d}_{21}(\mathrm{t})+\tau_{21}(\mathrm{t})\right) \mathrm{e}^{-\mathrm{v}}\right) \frac{\partial \mathrm{K}(\mathrm{u}, \mathrm{v} ; \mathrm{t})}{\partial \mathrm{v}} \\
& +\left(-\left(\mathrm{g}_{32}(\mathrm{t})+\mathrm{d}_{32}(\mathrm{t})+\tau_{32}(\mathrm{t})\right)+\left(\mathrm{d}_{32}(\mathrm{t})+\tau_{32}(\mathrm{t})\right) \mathrm{e}^{-\mathrm{v}}+\mathrm{g}_{32}(\mathrm{t}) \mathrm{e}^{\mathrm{v}}\right) \mathrm{K}(\mathrm{u}, \mathrm{v} ; \mathrm{t})
\end{aligned}
$$

By definition

$\frac{\partial \mathrm{K}(\mathrm{u}, \mathrm{v} ; \mathrm{t})}{\partial \mathrm{t}}=\mathrm{um}_{1,0}(\mathrm{t})+\mathrm{vm}_{0,1}(\mathrm{t})+\frac{1}{2} \mathrm{u}^{2} \mathrm{~m}_{2,0}(\mathrm{t})+\frac{1}{2} \mathrm{v}^{2} \mathrm{~m}_{0,2}(\mathrm{t})+\mathrm{uv} \mathrm{m}_{1,1}(\mathrm{t})+\ldots$

If we expand both side of the eqn. (9) in power of $u$ and $v$ and equate coefficient, We obtain the following system of differential equations,

$$
\begin{aligned}
& \frac{\mathrm{dm}_{1,0}(\mathrm{t})}{\mathrm{dt}}=\left(\mathrm{g}_{11}(\mathrm{t})-\tau_{11}(\mathrm{t})-\mathrm{d}_{11}(\mathrm{t})\right) \mathrm{m}_{1,0}(\mathrm{t}) \\
& \frac{\mathrm{dm}_{0,1}(\mathrm{t})}{\mathrm{dt}}=\tau_{11}(\mathrm{t}) \mathrm{m}_{1,0}(\mathrm{t})+\left(\mathrm{g}_{21}(\mathrm{t})-\mathrm{d}_{21}(\mathrm{t})-\tau_{21}(\mathrm{t})\right) \mathrm{m}_{0,1}(\mathrm{t}) \\
& \frac{\mathrm{dm}_{2,0}(\mathrm{t})}{\mathrm{dt}}=2\left(\mathrm{~g}_{11}(\mathrm{t})-\tau_{11}(\mathrm{t})-\mathrm{d}_{11}(\mathrm{t})\right) \mathrm{m}_{2,0}(\mathrm{t})+\left(\mathrm{g}_{11}(\mathrm{t})+\tau_{11}(\mathrm{t})+\mathrm{d}_{11}(\mathrm{t})\right) \mathrm{m}_{1,0}(\mathrm{t}) \\
& \frac{\mathrm{dm}_{0,2}(\mathrm{t})}{\mathrm{dt}}=\tau_{11}(\mathrm{t}) \mathrm{m}_{1,0}(\mathrm{t})+\left(\mathrm{g}_{21}(\mathrm{t})+\mathrm{d}_{21}(\mathrm{t})+\tau_{21}(\mathrm{t})\right) \mathrm{m}_{0,1}(\mathrm{t})+2\left(\mathrm{~g}_{32}(\mathrm{t})-\mathrm{d}_{21}(\mathrm{t})-\tau_{21}(\mathrm{t})\right) \mathrm{m}_{0,2}(\mathrm{t}) \\
& +2\left(\mathrm{~g}_{32}(\mathrm{t})-\mathrm{d}_{32}(\mathrm{t})-\tau_{32}(\mathrm{t})\right) \mathrm{m}_{0,1}(\mathrm{t})+\tau_{11}(\mathrm{t}) \mathrm{m}_{1,1}(\mathrm{t}) \\
& \frac{\mathrm{d} \mathrm{m}_{1,1}(\mathrm{t})}{\mathrm{dt}}=\left(\mathrm{g}_{11}(\mathrm{t})-\tau_{11}(\mathrm{t})-\mathrm{d}_{11}(\mathrm{t})\right) \mathrm{m}_{1,1}(\mathrm{t})+2 \tau_{11}(\mathrm{t}) \mathrm{m}_{2,0}(\mathrm{t})-\tau_{11}(\mathrm{t}) \mathrm{m}_{1,0}(\mathrm{t}) \\
& +\left(g_{21}(t)-d_{21}(t)-\tau_{21}(t)\right) m_{1,1}(t)+\left(g_{32}(t)-d_{32}(t)-\tau_{32}(t)\right) m_{1,0}(t)
\end{aligned}
$$

where $m_{i, j}(t)$ denotes the statistical moments of $\operatorname{order}(i, j)$ representing the number of normal cells and number of malignant cells in an organ at time $t$. The above system of ordinary differential equations is solved numerically using mathematica and results obtained are presented below. The differential equations are in terms of first and second order moments, which are presented for fixed values of parameters such as $\mathrm{g}_{11}=0.00001 ; \mathrm{d}_{11}=0.00001 ; \tau_{11}=0.3 ; \mathrm{g}_{21}=0.8 ; \mathrm{d}_{21}=1.0 \times 10^{-7} ; \tau_{21}=0.005$; $\mathrm{g}_{32}=0.15 ; \mathrm{d}_{32}=0.000001 ; \tau_{32}=1.0 \times 10^{-7}$ over a period of time. 


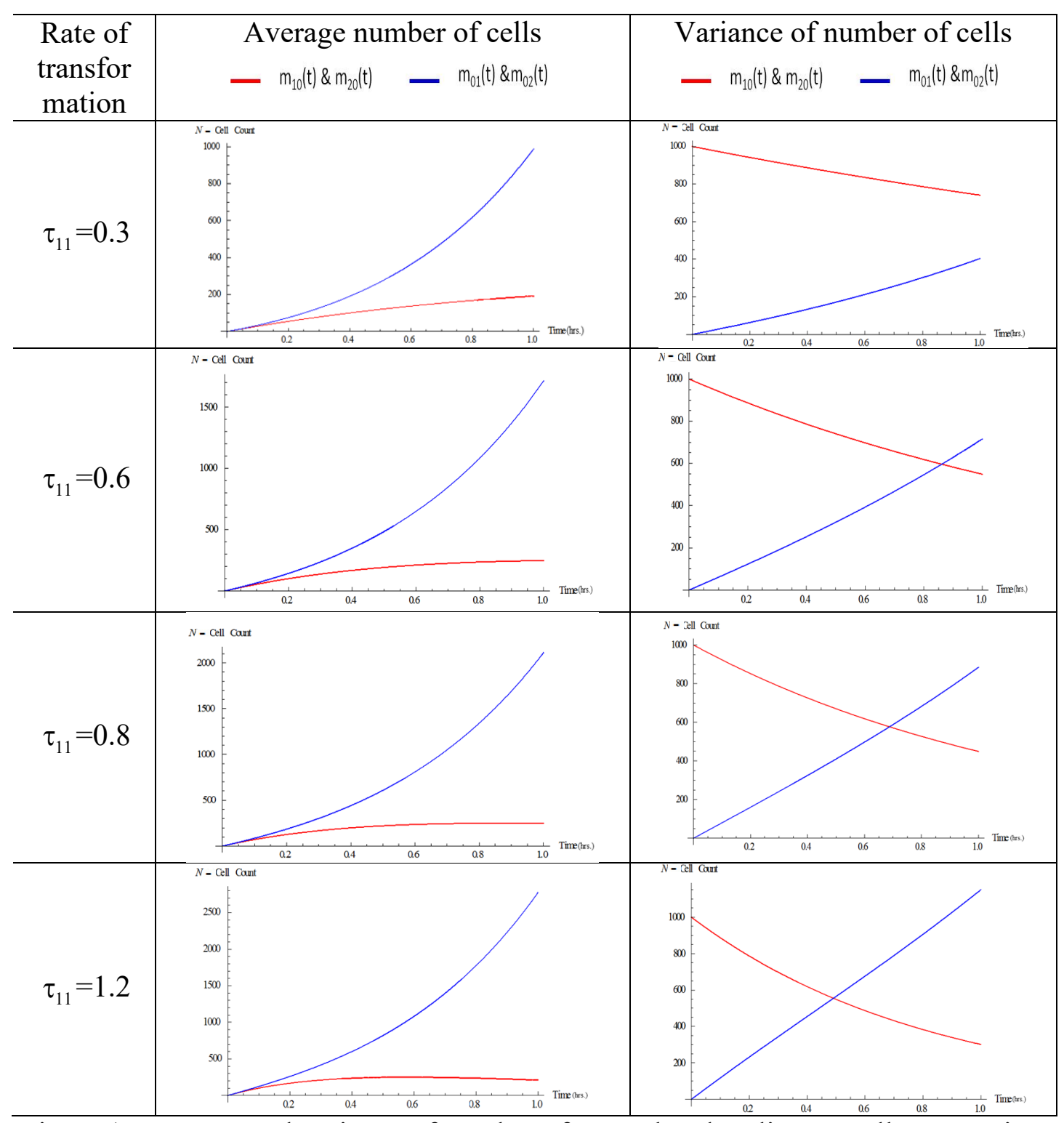

Figure 1: Average and variance of number of normal and malignant cells over a time

From Figure-1, it is representing the average number of normal cells, average number of malignant cells, variance of number of normal cells and variance of number of malignant cells in an organ over a period with the fixed parameter values and changing value of transformation rate of normal cells $\left(\tau_{11}\right)$.

The explicit expression for moments of the above model is derived under homogeneous processes by Rao et al., (2014) are

$$
\begin{aligned}
& \mathrm{m}_{1,0}(\mathrm{t})=\mathrm{N}_{0} \mathrm{e}^{\mathrm{At}} \\
& \mathrm{m}_{0,1}(\mathrm{t})=\frac{\tau_{11} \mathrm{~N}_{0} \mathrm{e}^{\mathrm{At}}}{\mathrm{A}-\mathrm{B}}+\left(\mathrm{M}_{0}-\frac{\tau_{11} \mathrm{~N}_{0}}{\mathrm{~A}-\mathrm{B}}\right) \mathrm{e}^{\mathrm{Bt}} \\
& \mathrm{m}_{2,0}(\mathrm{t})=\frac{\mathrm{DN} \mathrm{e}^{\mathrm{At}}}{\mathrm{A}}\left(\mathrm{e}^{\mathrm{At}}-1\right)
\end{aligned}
$$




$$
\begin{aligned}
\mathrm{m}_{0,2}(\mathrm{t})= & \frac{\tau_{11} \mathrm{~N}_{0} \mathrm{e}^{\mathrm{At}}}{(\mathrm{A}-2 \mathrm{~B})}+(\mathrm{F}+2 \mathrm{E})\left\{\frac{\tau_{11} \mathrm{~N}_{0} \mathrm{e}^{\mathrm{At}}}{(\mathrm{A}-2 \mathrm{~B})(\mathrm{A}-\mathrm{B})}-\left(\mathrm{M}_{0}-\frac{\tau_{11} \mathrm{~N}_{0}}{(\mathrm{~A}-\mathrm{B})}\right) \frac{\mathrm{e}^{\mathrm{Bt}}}{\mathrm{B}}\right\} \\
& +\tau_{11}\left\{\begin{array}{l}
\frac{\tau_{11} \mathrm{~N}_{0} \mathrm{D}}{\mathrm{A}}\left(\frac{\mathrm{e}^{2 \mathrm{At}}}{2(\mathrm{~A}-\mathrm{B})^{2}}+\frac{\mathrm{e}^{\mathrm{At}}}{(\mathrm{A}-2 \mathrm{~B}) \mathrm{B}}\right)-\frac{\left(\mathrm{E}-\tau_{11}\right) \mathrm{N}_{0} \mathrm{e}^{\mathrm{At}}}{(\mathrm{A}-2 \mathrm{~B}) \mathrm{B}} \\
-\left(\frac{\delta_{11} \mathrm{~N}_{0} \mathrm{D}-(\mathrm{A}-\mathrm{B})\left(\mathrm{E}-\delta_{11}\right) \mathrm{N}_{0}}{(\mathrm{~A}-\mathrm{B}) \mathrm{B}}\right) \frac{\mathrm{e}^{(\mathrm{A}-\mathrm{B}) \mathrm{t}}}{(\mathrm{A}-\mathrm{B})}
\end{array}\right\} \\
& -\left\{\begin{array}{l}
\frac{\tau_{11} \mathrm{~N}_{0}}{(\mathrm{~A}-2 \mathrm{~B})}+(\mathrm{F}+2 \mathrm{E})\left(\frac{\tau_{11} \mathrm{~N}_{0}-(\mathrm{A}-2 \mathrm{~B}) \mathrm{M}_{0}}{2(\mathrm{~A}-2 \mathrm{~B})(\mathrm{A}-\mathrm{B})^{2} \mathrm{~B}}\right) \\
+\tau_{11}^{2} \mathrm{~N}_{0}\left(\frac{2(\mathrm{~A}-\mathrm{B})\left(\delta_{11}-\mathrm{E}\right)+\mathrm{D} \tau_{11}}{2(\mathrm{~A}-2 \mathrm{~B})(\mathrm{A}-\mathrm{B})^{2}}\right)
\end{array}\right\} \mathrm{e}^{2 \mathrm{Bt}} \\
\mathrm{m}_{1,1}(\mathrm{t})= & \left\{\frac{\left.\left(\mathrm{E}-\tau_{11}\right) \mathrm{N}_{0} \mathrm{e}^{-\mathrm{Bt}}+\frac{\tau_{11} \mathrm{DN} \mathrm{N}_{0}}{\mathrm{~A}}\left(\frac{\mathrm{e}^{2 \mathrm{At}}}{(\mathrm{A}-\mathrm{B})}+\frac{\mathrm{e}^{\mathrm{At}}}{\mathrm{B}}\right)\right\}}{(\mathrm{B}-\mathrm{B}) \mathrm{B}}\right. \\
& +\left\{\frac{\tau_{11} \mathrm{DN}_{0}-(\mathrm{A}-\mathrm{B})\left(\mathrm{E}-\tau_{11}\right) \mathrm{N}_{0}}{(\mathrm{~A}+\mathrm{B}) \mathrm{t}}\right.
\end{aligned}
$$

where

$\mathrm{A}=\beta_{11}-\tau_{11}-\delta_{11} \quad \mathrm{~B}=\beta_{21}-\delta_{21}-\tau_{21} \quad \mathrm{D}=\beta_{11}+\tau_{11}+\delta_{11} \quad \mathrm{E}=\beta_{32}-\delta_{32}-\tau_{32}$

$\mathrm{F}=\beta_{21}+\delta_{21}+\tau_{21}$

$\mathrm{m}_{1,0}(\mathrm{t}), \mathrm{m}_{0,1}(\mathrm{t}), \mathrm{m}_{2,0}(\mathrm{t}), \mathrm{m}_{2,0}(\mathrm{t}), \mathrm{m}_{0,2}(\mathrm{t}), \mathrm{m}_{1,1}(\mathrm{t})$ are expected number of normal cells, expected number of malignant cells, variance of number of normal cells, variance of number of malignant cells, covariance of number of normal cells. Hence, the characteristics of the model are obtained through the moments which are obtained above.

\section{Limiting Behaviour of the Moments}

The limiting behaviour of the derived moments are obtained easily by taking limit $t \rightarrow \infty$. In particular the study on the normal cells and malignant cells in the cell's population, obviously our interest is to find the asymptotic performance of the ratio of the average (expected) number of normal cells and average (expected) number of malignant cells in the population (Bharucha-Reid, 1960).

Let $\mathrm{R}_{\mathrm{E}_{1}}(\mathrm{t})=\frac{\mathrm{m}_{1,0}(\mathrm{t})}{\mathrm{m}_{0,1}(\mathrm{t})}=\left\{\left[\mathrm{N}_{0} \mathrm{e}^{\mathrm{At}}\right] /\left[\frac{\tau_{11} \mathrm{~N}_{0} \mathrm{e}^{\mathrm{At}}}{\mathrm{A}-\mathrm{B}}+\left(\mathrm{M}_{0}-\frac{\tau_{11} \mathrm{~N}_{0}}{\mathrm{~A}-\mathrm{B}}\right) \mathrm{e}^{\mathrm{Bt}}\right]\right\}$

When $\mathrm{N}_{0}=1$ and $\mathrm{M}_{0}=0$, the ratio is reduced to

$\mathrm{R}_{\mathrm{E}_{1}}=\frac{(\mathrm{A}-\mathrm{B}) \mathrm{e}^{\mathrm{At}}}{\tau_{11}\left(\mathrm{e}^{\mathrm{At}}-\mathrm{e}^{\mathrm{Bt}}\right)}$

We observe that in the case of $(\mathrm{A}-\mathrm{B})>0$

$\lim _{t \rightarrow \infty} R_{E_{1}}(t)=\frac{(A-B)}{\tau_{11}}$ 
$\lim _{t \rightarrow \infty} R_{E_{1}}(t)=\frac{\left[\left(\beta_{11}-\tau_{11}-\delta_{11}\right)-\left(\beta_{21}-\delta_{21}-\tau_{21}\right)\right]}{\tau_{11}}$

If $\tau_{11}=\tau_{21}$ and $\delta_{11}=\delta_{21}$, then the above equation is reduced to

$\lim _{t \rightarrow \infty} R_{E_{1}}(t)=\frac{\left(\beta_{11}-\beta_{21}\right)}{\tau_{11}}$ (or) $\frac{\left(\beta_{11}-\beta_{21}\right)}{\tau_{21}}$

\section{Solution for the Model}

Let $\phi(x, y ; t)=\sum_{n=0}^{\infty} \sum_{m=0}^{\infty} p_{n, m}(t) x^{n} y^{m}$ is the joint probability generating function with respect to ' $n$ ' number of normal cells and ' $m$ ' number of malignant cells.

The partial differential equation governing the process is

$$
\begin{aligned}
& \frac{\partial}{\partial \mathrm{t}} \phi(\mathrm{x}, \mathrm{y} ; \mathrm{t})=\left\{-\left(\beta_{11}+\tau_{11}+\delta_{11}\right) \mathrm{x}+\delta_{11}+\beta_{11} \mathrm{x}^{2}+\tau_{11} \mathrm{y}\right\} \frac{\partial}{\partial \mathrm{x}} \phi(\mathrm{x}, \mathrm{y} ; \mathrm{t}) \\
&+\left\{-\left(\beta_{21}+\delta_{21}+\tau_{21}\right) \mathrm{y}+\beta_{21} \mathrm{y}^{2}+\left(\delta_{21}+\tau_{21}\right)\right\} \frac{\partial}{\partial \mathrm{y}} \phi(\mathrm{x}, \mathrm{y} ; \mathrm{t}) \\
&+\left\{-\left(\beta_{32}+\delta_{32}+\tau_{32}\right)+\left(\delta_{32}+\tau_{32}\right) \mathrm{y}^{-1}+\beta_{32} \mathrm{y}\right\} \phi(\mathrm{x}, \mathrm{y} ; \mathrm{t})
\end{aligned}
$$

with initial condition $\phi(\mathrm{x}, \mathrm{y} ; 0)=\mathrm{x}^{\mathrm{N}_{0}} \mathrm{y}^{\mathrm{M}_{0}}$; where $\mathrm{N}_{0}$ and $\mathrm{M}_{0}$ are the initial number of normal and malignant cells respectively. Using the Lagrange method of solution, the above equation has auxiliary equations

$$
\begin{aligned}
& \frac{\mathrm{dt}}{\underset{\text { (i) }}{1}}=\frac{-\mathrm{dx}}{\left\{-\left(\beta_{11}+\tau_{11}+\delta_{11}\right) \mathrm{x}+\delta_{11}+\beta_{11} \mathrm{x}^{2}+\tau_{11} \mathrm{y}\right\}}=\frac{-\mathrm{dy}}{\left\{-\left(\beta_{21}+\delta_{21}+\tau_{21}\right) \mathrm{y}+\beta_{21} \mathrm{y}^{2}+\left(\delta_{21}+\tau_{21}\right)\right\}} \\
& =\frac{\mathrm{d} \phi}{\phi\left\{-\left(\beta_{32}+\delta_{32}+\tau_{32}\right)+\left(\delta_{32}+\tau_{32}\right) \mathrm{y}^{-1}+\beta_{32} \mathrm{y}\right\}}
\end{aligned}
$$

From (i) and (iii) becomes

$$
\begin{aligned}
& \frac{\mathrm{dt}}{1}=\frac{-\mathrm{dy}}{\left\{-\left(\beta_{21}+\delta_{21}+\tau_{21}\right) \mathrm{y}+\beta_{21} \mathrm{y}^{2}+\left(\delta_{21}+\tau_{21}\right)\right\}} \\
& \frac{\mathrm{dt}}{1}=\frac{\mathrm{dy}}{\left(\beta_{21} \mathrm{y}-\left(\delta_{21}+\tau_{21}\right)\right)(\mathrm{y}-1)}
\end{aligned}
$$

Integrating (28) we get

$$
\begin{aligned}
& \mathrm{t}=\frac{1}{\left(\beta_{21}-\left(\delta_{21}+\tau_{21}\right)\right)} \log \left\{\frac{\left(\beta_{21} \mathrm{y}-\left(\delta_{21}+\tau_{21}\right)\right)}{(\mathrm{y}-1)}\right\}+\log \theta_{1} \\
& \Rightarrow \theta_{1}=\frac{\left(\beta_{21} \mathrm{y}-\left(\delta_{21}+\tau_{21}\right)\right)}{(\mathrm{y}-1)} \exp \left\{-\left(\left(\beta_{21}-\left(\delta_{21}+\tau_{21}\right)\right) \mathrm{t}\right)\right\}
\end{aligned}
$$

where $\theta_{1}$ is an integrating constant 
Consider (ii) and (iii)

$$
\begin{gathered}
\frac{-\mathrm{dx}}{\left\{-\left(\beta_{11}+\tau_{11}+\delta_{11}\right) \mathrm{x}+\delta_{11}+\beta_{11} \mathrm{x}^{2}+\tau_{11} \mathrm{y}\right\}}=\frac{-\mathrm{dy}}{\left\{-\left(\beta_{21}+\delta_{21}+\tau_{21}\right) \mathrm{y}+\beta_{21} \mathrm{y}^{2}+\left(\delta_{21}+\tau_{21}\right)\right\}} \\
\frac{\mathrm{dx}}{\mathrm{dy}}=\frac{\beta_{11} \mathrm{x}^{2}}{\left\{-\left(\beta_{21}+\delta_{21}+\tau_{21}\right) \mathrm{y}+\beta_{21} \mathrm{y}^{2}+\left(\delta_{21}+\tau_{21}\right)\right\}}+\frac{-\left(\beta_{11}+\tau_{11}+\delta_{11}\right) \mathrm{x}}{\left\{-\left(\beta_{21}+\delta_{21}+\tau_{21}\right) \mathrm{y}+\beta_{21} \mathrm{y}^{2}+\left(\delta_{21}+\tau_{21}\right)\right\}} \\
\quad+\frac{\left\{\delta_{11}+\tau_{11} \mathrm{y}\right\}}{\left\{-\left(\beta_{21}+\delta_{21}+\tau_{21}\right) \mathrm{y}+\beta_{21} \mathrm{y}^{2}+\left(\delta_{21}+\tau_{21}\right)\right\}}
\end{gathered}
$$

which is in the form of Riccati first order ordinary differential equation, $\mathrm{x}=\mathrm{y}$ is the particular solution, and by substitution of $\mathrm{x}=\mathrm{y}+\left(1 / \mathrm{v}^{*}(\mathrm{y})\right)$, it leads to

$$
\begin{aligned}
& \frac{d}{d y} v^{*}(y)+\left\{\frac{2 y \beta_{11}-\left(\beta_{11}+\tau_{11}+\delta_{11}\right)}{\left\{-\left(\beta_{21}+\delta_{21}+\tau_{21}\right) y+\beta_{21} y^{2}+\left(\delta_{21}+\tau_{21}\right)\right\}}\right\} v^{*}(y) \\
& +\frac{\beta_{11}}{\left\{-\left(\beta_{21}+\delta_{21}+\tau_{21}\right) y+\beta_{21} y^{2}+\left(\delta_{21}+\tau_{21}\right)\right\}}=0 \\
& \Rightarrow \frac{d}{d y} v^{*}(y)+\left\{\frac{2 y \beta_{11}-\left(\beta_{11}+\tau_{11}+\delta_{11}\right)}{\left(\beta_{21} y-\left(\delta_{21}+\tau_{21}\right)\right)(y-1)}\right\} v^{*}(y)+\frac{\beta_{11}}{\left(\beta_{21} y-\left(\delta_{21}+\tau_{21}\right)\right)(y-1)}=0
\end{aligned}
$$

It reduces to

$$
\begin{aligned}
\mathrm{v}^{*}(\mathrm{y})= & \frac{\beta_{11} \beta_{21}}{\beta_{21}\left(\tau_{11}+\delta_{11}\right)+\beta_{11}\left(\beta_{21}-2\left(\delta_{21}+\tau_{21}\right)\right)} \\
& \text { Hypergeometric } 2 \mathrm{~F}_{1}\left[1, \frac{2 \beta_{11}}{\beta_{21}}, 1+\frac{2 \beta_{11}}{\beta_{21}}-\frac{\left(-\beta_{11}+\tau_{11}+\delta_{11}\right)}{-\beta_{21}+\tau_{21}+\delta_{21}},-\frac{\left.-\beta_{21} \mathrm{y}+\tau_{21}+\delta_{21}\right]}{-\beta_{21}+\tau_{21}+\delta_{21}}\right] \\
& +\theta_{2}(\mathrm{y}-1)^{-\frac{\left(-\beta_{11}+\tau_{11}+\delta_{11}\right)}{-\beta_{21}+\tau_{21}+\delta_{21}}}\left(\beta_{21} \mathrm{y}-\left(\delta_{21}+\tau_{21}\right)\right)^{-\frac{2 \beta_{11}}{\beta_{21}}}-\frac{\left(-\beta_{11}+\tau_{11}+\delta_{11}\right)}{-\beta_{21}+\tau_{21}+\delta_{21}} \\
\mathrm{v}^{*}(\mathrm{y})= & \zeta(\mathrm{y})+\theta_{1}^{*}(\mathrm{y}-1)^{-\frac{\left(-\beta_{11}+\tau_{11}+\delta_{11}\right)}{-\beta_{21}+\tau_{21}+\delta_{21}}}\left(\beta_{21} \mathrm{y}-\left(\delta_{21}+\tau_{21}\right)\right)^{-\frac{2 \beta_{11} 1}{\beta_{21}}-\frac{\left(-\beta_{11}+\tau_{11}+\delta_{11}\right)}{-\beta_{21}+\tau_{21}+\delta_{21}}}
\end{aligned}
$$

where

$$
\begin{aligned}
\zeta(\mathrm{y})= & \frac{\beta_{11} \beta_{21}}{\beta_{21}\left(\tau_{11}+\delta_{11}\right)+\beta_{11}\left(\beta_{21}-2\left(\delta_{21}+\tau_{21}\right)\right)} \\
& \text { Hypergeometric } 2 \mathrm{~F}_{1}\left[1, \frac{2 \beta_{11}}{\beta_{21}}, 1+\frac{2 \beta_{11}}{\beta_{21}}-\frac{\left(-\beta_{11}+\tau_{11}+\delta_{11}\right)}{-\beta_{21}+\tau_{21}+\delta_{21}},-\frac{-\beta_{21} \mathrm{y}+\tau_{21}+\delta_{21}}{-\beta_{21}+\tau_{21}+\delta_{21}}\right]
\end{aligned}
$$

$\theta_{2}$ is the integrating constant and for arbitrary $\mathrm{a}, \mathrm{b}$, c and real $-1<\mathrm{z}<1$,

$$
2 \mathrm{~F}_{1}(\mathrm{a}, \mathrm{b}, \mathrm{c} ; \mathrm{z})=\frac{\Gamma(\mathrm{c})}{\Gamma(\mathrm{b}) \Gamma(\mathrm{c}-\mathrm{b})} \int_{0}^{1} \frac{\mathrm{t}^{\mathrm{b}-1}(1-\mathrm{t})^{(\mathrm{c}-\mathrm{b}-1)}}{(1-\mathrm{tz})^{\mathrm{a}}} \mathrm{dt}
$$

From (i) and (iv),

$$
\begin{aligned}
& \frac{\mathrm{dt}}{1}=\frac{\mathrm{d} \phi}{\phi\left\{-\left(\beta_{32}+\delta_{32}+\tau_{32}\right)+\left(\delta_{32}+\tau_{32}\right) \mathrm{y}^{-1}+\beta_{32} \mathrm{y}\right\}} \\
& \Rightarrow \frac{\mathrm{dt}}{1}=\frac{\mathrm{yd} \phi}{\phi\left\{-\left(\beta_{32}+\delta_{32}+\tau_{32}\right) \mathrm{y}+\left(\delta_{32}+\tau_{32}\right)+\beta_{32} \mathrm{y}^{2}\right\}} \\
& \Rightarrow \exp \left(\mathrm{t}\left\{-\left(\beta_{32}+\delta_{32}+\tau_{32}\right) \mathrm{y}+\left(\delta_{32}+\tau_{32}\right)+\beta_{32} \mathrm{y}^{2}\right\} \mathrm{y}^{-1}\right) * \phi^{-1}=\theta_{3}
\end{aligned}
$$


Let us suppose that at $\mathrm{t}=0$, the function $\phi(\mathrm{x}, \mathrm{y} ; 0)=\mathrm{x}^{\mathrm{N}_{0}} \mathrm{y}^{\mathrm{M}_{0}}$. By assuming that there are ' $\mathrm{N}_{0}$ ' number of normal cells and ' $\mathrm{M}_{0}$ ' number of malignant cells at initial $\mathrm{t}=0$.

The general solution for the model is

$$
\begin{aligned}
& \phi(\mathrm{x}, \mathrm{y} ; 0)=\mathrm{x}^{\mathrm{N}_{0}} \mathrm{y}^{\mathrm{M}_{0}} \\
& =\psi\left(\left(\mathrm{v}^{*}(\mathrm{y})-\zeta(\mathrm{y})\right)\right. \\
& \left.\left((\mathrm{y}-1)^{-\frac{\left(-\beta_{11}+\tau_{11}+\delta_{11}\right)}{-\beta_{21}+\tau_{21}+\delta_{21}}}\left(\beta_{21} \mathrm{y}-\left(\delta_{21}+\tau_{21}\right)\right)^{-\frac{2 \beta_{11}}{\beta_{21}}-\frac{\left(-\beta_{11}+\tau_{11}+\delta_{11}\right)}{-\beta_{21}+\tau_{21}+\delta_{21}}}\right)^{-1}, \frac{\left(\beta_{21} \mathrm{y}-\left(\delta_{21}+\tau_{21}\right)\right)}{(\mathrm{y}-1)}\right)
\end{aligned}
$$

Elimination of the integration constants, we get

$$
\begin{gathered}
\phi(\mathrm{x}, \mathrm{y} ; \mathrm{t})=\left(\frac{\left(\delta_{21}+\tau_{21}\right)+\mathrm{ze}^{-\mathrm{bt}}}{\beta_{21}+\mathrm{ze}^{-\mathrm{bt}}}\right)^{\mathrm{M}_{0}}\left[\frac{\left(\delta_{21}+\tau_{21}\right)+\mathrm{ze}^{-\mathrm{bt}}}{\beta_{21}+\mathrm{ze}^{-\mathrm{bt}}}+\left[\frac{\left(\delta_{21}+\tau_{21}\right)+\mathrm{ze}^{-\mathrm{bt}}}{\beta_{21}+\mathrm{ze}^{-\mathrm{bt}}} \zeta\left(\mathrm{ze}^{-\mathrm{bt}}\right)\right.\right. \\
+\left(\frac{1}{\mathrm{x}-\mathrm{y}}-\zeta(\mathrm{y})\right)\left(\frac{\beta_{21}+\mathrm{z}}{\beta_{21}+\mathrm{ze}^{-\mathrm{bt}}}\right){ }^{-\left(\frac{\left(-\beta_{11}+\tau_{11}+\delta_{11}\right)}{-\beta_{21}+\tau_{21}+\delta_{21}}+\frac{2 \beta_{11}}{\beta_{21}}-\frac{\left(-\beta_{11}+\tau_{11}+\delta_{11}\right)}{-\beta_{21}+\tau_{21}+\delta_{21}}\right)} \\
\left.\left.\exp \left(\frac{\left(-\beta_{11}+\tau_{11}+\delta_{11}\right)}{-\beta_{21}+\tau_{21}+\delta_{21}} \mathrm{bt}\right)\right]^{-1}\right]^{\mathrm{N}_{0}}
\end{gathered}
$$

This is similar to the model developed by Birkhead (1986), the form obtained in (35) can be used to find the probability that there being no malignant cell at time $t$ after the start of the process is $\phi(1,0 ; \mathrm{t})$. For given probability generating function, the survival function of the time to the appearance of the first intermediate cell and first malignant cell in the population of cells $S(t)$, $\phi(1,0, t)=\sum_{\mathrm{i}} \mathrm{p}_{\mathrm{i}, 0}(\mathrm{t})=\mathrm{P}(\mathrm{T}>\mathrm{t})=\mathrm{S}(\mathrm{t})$ the corresponding hazard functions are $\mathrm{h}(\mathrm{t})=-\frac{\phi^{\prime}(1,0, \mathrm{t})}{\phi(1,0, \mathrm{t})}$.

On solving $\phi(1,0, t)$ and derivative of them with respect to $t$, this enables us to find survival and hazard functions.

\section{Non-homogeneous Processes for Cell Growths with Regression Approach}

The mutation parameter $\tau_{11}(t)$ is time - dependent function (it is a function of time $t$ ). The migration parameter $\tau_{21}(\mathrm{t}), \tau_{32}(\mathrm{t})$ is also function of time $\mathrm{t}$. If we assume the mutation and migration parameters are constant then it is very simple to derive the statistical moment from the above system of linear differential equations. If $\tau_{21}(t)=\tau_{32}(t)=0$, then model deduced to the simple two state models with single mutation, which means that there is no migration taken place. (Serio, 1986) the statistical moments for the normal cells and malignant cells were derived in the form of system of linear differential equation with non-homogeneous parameters. Let us consider $\tau_{21}(t)=\tau_{32}(t)=0$ and $g_{32}(t)=d_{32}(t)=0$ i.e., there is no migration is take place. Then, the system of linear differential equations becomes

$\frac{\mathrm{d} \mathrm{m}_{1,0}(\mathrm{t})}{\mathrm{dt}}=\left(\mathrm{g}_{11}(\mathrm{t})-\tau_{11}(\mathrm{t})-\mathrm{d}_{11}(\mathrm{t})\right) \mathrm{m}_{1,0}(\mathrm{t})$ 


$$
\begin{aligned}
& \frac{\mathrm{d} \mathrm{m}_{0,1}(\mathrm{t})}{\mathrm{dt}}=\tau_{11}(\mathrm{t}) \mathrm{m}_{1,0}(\mathrm{t})+\left(\mathrm{g}_{21}(\mathrm{t})-\mathrm{d}_{21}(\mathrm{t})\right) \mathrm{m}_{0,1}(\mathrm{t}) \\
& \frac{\mathrm{dm}_{2,0}(\mathrm{t})}{\mathrm{dt}}=2\left(\mathrm{~g}_{11}(\mathrm{t})-\tau_{11}(\mathrm{t})-\mathrm{d}_{11}(\mathrm{t})\right) \mathrm{m}_{2,0}(\mathrm{t})+\left(\mathrm{g}_{11}(\mathrm{t})+\tau_{11}(\mathrm{t})+\mathrm{d}_{11}(\mathrm{t})\right) \mathrm{m}_{1,0}(\mathrm{t}) \\
& \frac{\mathrm{d \textrm {m } _ { 0 , 2 }}(\mathrm{t})}{\mathrm{dt}}=\tau_{11}(\mathrm{t}) \mathrm{m}_{1,0}(\mathrm{t})+\left(\mathrm{g}_{21}(\mathrm{t})+\mathrm{d}_{21}(\mathrm{t})\right) \mathrm{m}_{0,1}(\mathrm{t})+2\left(\mathrm{~g}_{21}(\mathrm{t})-\mathrm{d}_{21}(\mathrm{t})\right) \mathrm{m}_{0,2}(\mathrm{t})+\tau_{11}(\mathrm{t}) \mathrm{m}_{1,1}(\mathrm{t}) \\
& \frac{\mathrm{dm}_{1,1}(\mathrm{t})}{\mathrm{dt}}=\left(\left\{\left(\mathrm{g}_{11}(\mathrm{t})+\mathrm{g}_{21}(\mathrm{t})\right)-\left(\mathrm{d}_{11}(\mathrm{t})+\mathrm{d}_{21}(\mathrm{t})\right)\right\}-\tau_{11}(\mathrm{t})\right) \mathrm{m}_{1,1}(\mathrm{t})+2 \tau_{11}(\mathrm{t}) \mathrm{m}_{2,0}(\mathrm{t})-\tau_{11}(\mathrm{t}) \mathrm{m}_{1,0}(\mathrm{t})
\end{aligned}
$$

For any organ, it contains active cells (living cells) and inactive cells (benign and necrosis), active cells are participating in the growth and inactive cells are not participating in the growth. Every cells in has the life span and ultimate get died, and then it is replace by the new one. Therefore in the growth process death also to be considered. The normal active cells are having a deterministic growth whereas abnormal active cells do not under a deterministic growth. If it is assumed that the growth of normal cells and malignant cells are deterministic and exponential (Armitage, 1960). Then, the expected number of cells contributed by normal cell to the cell population is

$$
\mathrm{m}_{1,0}(\mathrm{t})=\exp \left\{\int_{0}^{\mathrm{t}}\left\{\left(\mathrm{g}_{11}(\xi)-\mathrm{d}_{11}(\xi)\right)+\tau_{11}(\xi)\right\} \mathrm{d} \xi\right\}
$$

The expected number of cells contributed by malignant cells to the cell population is

$$
\mathrm{m}_{0,1}(\mathrm{t})=\exp \left\{\int_{0}^{\mathrm{t}}\left\{\left(\mathrm{g}_{21}(\xi)-\mathrm{d}_{21}(\xi)\right)\right\} \mathrm{d} \xi\right\}
$$

The expected total number of cells in an organ for a given time $t$ is defined as

$$
\begin{aligned}
& \mathrm{E}[\mathfrak{I}(\mathrm{t})]=\mathrm{E}[\mathrm{X}(\mathrm{t})]+\mathrm{E}[\mathrm{Y}(\mathrm{t})]=\mathrm{m}_{1,0}(\mathrm{t})+\mathrm{m}_{0,1}(\mathrm{t}) \\
& \mathrm{E}[\mathfrak{I}(\mathrm{t})]=\exp \left\{\int_{0}^{\mathrm{t}}\left\{\left(\mathrm{g}_{11}(\xi)-\mathrm{d}_{11}(\xi)\right)+\tau_{11}(\xi)\right\} \mathrm{d} \xi\right\}+\exp \left\{\int_{0}^{\mathrm{t}}\left\{\left(\mathrm{g}_{21}(\xi)-\mathrm{d}_{21}(\xi)\right)\right\} \mathrm{d} \xi\right\}
\end{aligned}
$$

Usually the normal cell population will give an impact upon the total number of cells and malignant cells. The normal cells population can be predicted, if the malignant cells population and total number of cells in an organ available through the relation of regression model as follows

$$
\mathrm{m}_{1,0}(\mathrm{t})=\exp \left\{\int_{0}^{\mathrm{t}}\left\{\left(\mathrm{g}_{11}(\xi)-\mathrm{d}_{11}(\xi)\right)+\tau_{11}(\xi)\right\} \mathrm{d} \xi\right\}+\exp \left\{\int_{0}^{\mathrm{t}}\left\{\left(\mathrm{g}_{21}(\xi)-\mathrm{d}_{21}(\xi)\right)\right\} \mathrm{d} \xi\right\}-\mathrm{m}_{0,1}(\mathrm{t})
$$

Substituting the above relation to the system of differential equations we have the following

$$
\begin{aligned}
\frac{\mathrm{d} \mathrm{m}_{1,1}(\mathrm{t})}{\mathrm{dt}}=\left(\left\{\left(\mathrm{g}_{11}(\mathrm{t})+\mathrm{g}_{21}(\mathrm{t})\right)-\left(\mathrm{d}_{11}(\mathrm{t})+\mathrm{d}_{21}(\mathrm{t})\right)\right\}-\tau_{11}(\mathrm{t})\right) \mathrm{m}_{1,1}(\mathrm{t})+2 \tau_{11}(\mathrm{t}) \mathrm{m}_{2,0}(\mathrm{t}) \\
-\tau_{11}(\mathrm{t})\left\{\exp \left\{\int_{0}^{\mathrm{t}}\left\{\left(\mathrm{g}_{11}(\xi)-\mathrm{d}_{11}(\xi)\right)+\tau_{11}(\xi)\right\} \mathrm{d} \xi\right\}+\exp \left\{\int_{0}^{\mathrm{t}}\left\{\left(\mathrm{g}_{21}(\xi)-\mathrm{d}_{21}(\xi)\right)\right\} \mathrm{d} \xi\right\}-\mathrm{m}_{0,1}(\mathrm{t})\right\} \\
\frac{\mathrm{dm}_{0,1}(\mathrm{t})}{\mathrm{dt}}=\tau_{11}(\mathrm{t})\left\{\exp \left\{\int_{0}^{\mathrm{t}}\left\{\left(\mathrm{g}_{11}(\xi)-\mathrm{d}_{11}(\xi)\right)+\tau_{11}(\xi)\right\} \mathrm{d} \xi\right\}\right. \\
\left.+\exp \left\{\int_{0}^{\mathrm{t}}\left\{\left(\mathrm{g}_{21}(\xi)-\mathrm{d}_{21}(\xi)\right)\right\} \mathrm{d} \xi\right\}-\mathrm{m}_{0,1}(\mathrm{t})\right\}+\left(\mathrm{g}_{21}(\mathrm{t})-\mathrm{d}_{21}(\mathrm{t})\right) \mathrm{m}_{0,1}(\mathrm{t})
\end{aligned}
$$




$$
\begin{aligned}
\frac{\mathrm{dm}_{0,2}(\mathrm{t})}{\mathrm{dt}}=\tau_{11}(\mathrm{t})\left\{\exp \left\{\int_{0}^{\mathrm{t}}\left\{\left(\mathrm{g}_{11}(\xi)-\mathrm{d}_{11}(\xi)\right)+\tau_{11}(\xi)\right\} \mathrm{d} \xi\right\}+\exp \left\{\int_{0}^{\mathrm{t}}\left\{\left(\mathrm{g}_{21}(\xi)-\mathrm{d}_{21}(\xi)\right)\right\} \mathrm{d} \xi\right\}-\mathrm{m}_{0,1}(\mathrm{t})\right\} \\
+\left(\mathrm{g}_{21}(\mathrm{t})+\mathrm{d}_{21}(\mathrm{t})\right) \mathrm{m}_{0,1}(\mathrm{t})+2\left(\mathrm{~g}_{21}(\mathrm{t})-\mathrm{d}_{21}(\mathrm{t})\right) \mathrm{m}_{0,2}(\mathrm{t})+\tau_{11}(\mathrm{t}) \mathrm{m}_{1,1}(\mathrm{t})
\end{aligned}
$$

The mathematical complexity in solving the above system of ordinary differential equations, numerically solved under homogeneous condition and presented the average number of malignant and variance of number of malignant cells at give time in the form of graphs as follows, $g_{11}=0.02$; $\mathrm{d}_{11}=0.001 ; \mathrm{g}_{21}=0.03 ; \mathrm{d}_{21}=0.00001 ; \tau_{11}=0.000025$. -
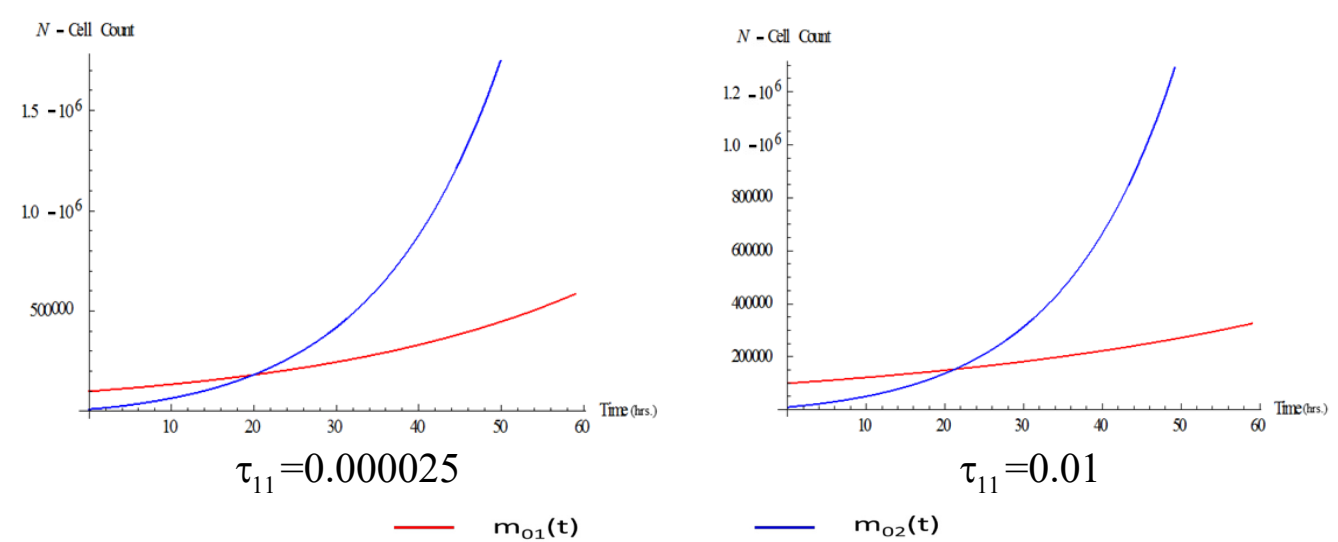

Figure 2: Average and variance of number of malignant cells

From Figure-2, it is representing the average number of malignant cells and variance of number of malignant cells in an organ over a period time with the fixed parameter values. The variance of number of cells is increased rapidly as compared to the average number of malignant cells over a period of time $t$ with given mutation rate.

\section{Conclusion}

This study can helps to quantification the growth of malignant cells under an abnormal and normal condition, and a proper understanding of growth of different type of cells over a period of time are more helpful in proper monitoring the patient's health and to apply optimum controlling strategies to control growth of cells and maintain the good health. This study has the limitation of obtaining real time data for analysing the model, but an appropriate data can be obtained from the clinical experimental laboratories.

\section{Acknowledgement}

I sincerely thank the reviewers for their valuable comments to improve the paper.

\section{References}

[1] G. Serio, Two-Stage Stochastic Model for Carcinogenesis with Time-Dependent Parameters, Statistics \& Probability Letters 2(1984) 95-103.

[2] W. Y. Tan, A stochastic model for the formation of metastatic foci at distant sites. Mathematical and Computer Modelling. 12(1989) 1093-1102.

[3] D. W. Quinn, The method of characteristics applied to a stochastic two-stage model of carcinogenesis. Mathematical and Computer Modelling. 25(1997) 1-13.

[4] S. H. Moolgavkar, D. J. Venzon, Two-event models for carcinogenesis: incidence curves for childhood and adult tumors. Mathematical Biosciences. 47(1979) 55-77. 
[5] W. Y. TAN,. A Nonhomogeneous Two-Stage Carcinogenesis Model, Mathl. Modelling. 9(1987) 631-642.

[6] P. Tirupathi Rao, J. Jayabharathiraj, B.N. Naveen Kumar, C. L. Usha, P. Rajasekhara Reddy, Stochastic Modelling of Tumor Growth within Organ during chemotherapy using Bivariate Birth, Death and Migration Processes, IOSR Journal of Mathematics. 10(2014) 01-08

[7] B. G. Birkhead, The Transient Solution of the Linear Birth-Death Process with Random Spontaneous Mutation, Mathematical Biosciences. 82(1986) 193-200.

[8] S. H. Moolgavkar, Model for human carcinogenesis: action of environmental agents, Environmental Health Perspectives. 50(1983) 285-91.

[9] B. P. Armitage, The Statistical theory of bacterial populations subject to mutation. Journal of the Royal Statistical Society, 14(1952) 1-40.

[10]A.T. Bharucha-Reid, Elements of the theory of markov processes and their applications, New York, McGraw-Hill, 1960. 